\title{
Informationen der Gesellschaft
}

\section{Vorstand}

Präsidentin

Frau Prof. Dr. Heide Siggelkow Universitätsmedizin Göttingen (UMG)

Georg-August-Universität

Robert-Koch-Str. 40

37075 Göttingen

Tel.: (+49) 551-63374622

Fax: (+49) 0551-53374646

E-Mail:

heide.siggelkow@endokrinologikum.com

\section{Vizepräsident}

Herr Prof. Dr. Hans-Christof Schober

Klinikum Südstadt Rostock

Südring 81

18059 Rostock

Tel: (+49) 0381-4401-5000

Fax: (+49) 0381-4401-5099

E-Mail:

Hans-christof.schober@kliniksued-rostock.de

\section{Kassenwart}

Dr. med. Winfried Demary

Bahnhofstr. 5

31134 Hildesheim

E-Mail: drdemary@gmx.de

\section{Schriftführerin}

Priv.-Doz. Dr. med. Gabriele Lehmann

Klinik Innere Medizin III

Am Klinikum 1

07747 Jena

Tel.: +49(0) 3641/93243-27

Fax: +49(0) 3641/93260-12

E-Mail: gabriele.lehmann@med.uni-jena.de

\section{DGO im Internet}

www.dgosteo.de

\section{Geschäftsstelle}

bei der Schriftführerin

Priv.-Doz. Dr. med. Gabriele Lehmann Klinik Innere Medizin III

Am Klinikum 1, 07747 Jena

Tel.: +49(0)3641/93243-27

Fax: $+49(0) 3641 / 93260-12$

E-Mail:

gabriele.lehmann@med.uni-jena.de

\section{Mitgliedsbeitrag}

Der Mitgliedsbeitrag 2018 beträgt für ordentliche Mitglieder Euro 80,-.

Der Beitrag schließt den Bezug der Fachzeitschrift Osteologie ein.

\section{Beitragskonto}

DGO e.V.

Sparkasse Göttingen

BLZ: 260500 01, Kontonummer: 56017163

IBAN: DE07260500010056017163

BIC (SWIFT): NOLADE21GOE

\section{Arbeitskreise}

\section{AK Diabetes und Knochen}

Sprecher:

Prof. Dr. med. Lorenz Hofbauer

Universitätsklinikum Dresden

Medizinische Klinik und Poliklinik III

Bereich Endokrinologie,

Diabetes \& Osteologie

Fetscherstr. 74, 01307 Dresden

Tel.: +49(0) 351/458-3173

Fax: $+49(0) 351 / 458-4309$

E-Mail:

lorenz.hofbauer@uniklinikum-dresden.de

und

Prof. Dr. med. Christian Meier

Facharzt FMH für Endokrinologie-

Diabetologie u. Innere Medizin

Universitätsspital Basel

Missionsstrasse 24, 4055 Basel, Schweiz

Te.l: $+41(0) 61 / 2649797$

Fax: $+41(0) 61 / 2649796$

E-Mail: christian.meier@unibas.ch

\section{AK Osteotechnologie}

Sprecher:

Univ.-Prof. Dr. rer. nat. Kay Raum Charité - Universitätsmedizin Berlin Julius Wolff Institute \& Berlin-Brandenburg School for Regenerative Therapies Augustenburger Platz 1, 13353 Berlin Tel.: +49 (0) 30/450-539503

Fax: $+49(0) 30 / 450-539952$

E-Mail: kay.raum@charite.de
Impressum

Verantwortlich für den Inhalt "DGO - Informationen der Gesellschaft" Priv.-Doz. Dr. Gabriele Lehmann, Jena 